Report No.: ORNL/TM-2002/20

\title{
PLUTONIUM ATTRIBUTE ESTIMATION FROM PASSIVE NMIS MEASUREMENTS AT VNIIEF
}

\author{
J. K. Mattingly
}

January 2002

\author{
Prepared by the \\ Oak Ridge National Laboratory \\ Oak Ridge, Tennessee 37831 \\ managed by \\ UT-Battelle, LLC \\ for \\ U.S. Department of Energy \\ under contract DE-AC05-00OR22725
}




\section{DISCLAIMER}

This report was prepared as an account of work sponsored by an agency of the United States government. Neither the United States Government nor any agency thereof, nor any of their employees, makes any warranty, express or implied, or assumes any legal liability or responsibility for the accuracy, completeness, or usefulness of any information, apparatus, product, or process disclosed, or represents that its use would not infringe privately owned rights. Reference herein to any specific commercial product, process, or service by trade name, trademark, manufacturer, or otherwise, does not necessarily constitute or imply its endorsement, recommendation, or favoring by the United States Government or any agency thereof. The views and opinions of authors expressed herein do not necessarily state or reflect those of the United States Government or any agency thereof. 


\section{PLUTONIUM ATTRIBUTE ESTIMATION FROM PASSIVE NMIS MEASUREMENTS AT VNIIEF}

Currently, the most relevant application of NMIS for plutonium attribute estimation stems from measurements performed jointly by Oak Ridge National Laboratory (ORNL) and Russian Federal Nuclear Center, All-Russia Scientific Research Institute of Experimental Physics (RFNC-VNIIEF) personnel at RFNC-VNIIEF facilities in Sarov, Russia in June and July 2000.

During these measurements at VNIIEF NMIS was applied in its passive mode to eight unclassified plutonium spherical shells. ${ }^{1}$ The shells' properties spanned the following ranges:

- Composition: $\delta$-phase plutonium-metal, constant

- Relative ${ }^{240} \mathrm{Pu}$-content $\left(f_{240 P u}\right): f_{240 P u}=1.77 \%\left\{\mathrm{~g}{ }^{240} \mathrm{Pu} / \mathrm{g} \mathrm{Pu}\right\}$, constant

- Inner radius $\left(r_{1}\right): 10.0 \mathrm{~mm} \leq r_{1} \leq 53.5 \mathrm{~mm}$, mean $r_{1}=33.5 \mathrm{~mm}$

- Outer radius $\left(r_{2}\right): 31.5 \mathrm{~mm} \leq r_{2} \leq 60.0 \mathrm{~mm}$, mean $r_{2}=46.6 \mathrm{~mm}$

- Radial thickness $(\Delta r): 6.4 \mathrm{~mm} \leq \Delta r \leq 30.2 \mathrm{~mm}$, mean $\Delta r=13.1 \mathrm{~mm}$

- Plutonium mass $\left(m_{P u}\right): 1829 \mathrm{~g} \leq m_{P u} \leq 4468 \mathrm{~g}$, mean $m_{P u}=3265 \mathrm{~g}$

The features of these measurements were analyzed to extract the attributes of each plutonium shell. Given that the samples measured were of constant composition, geometry, and relative ${ }^{240} \mathrm{Pu}$-content, each shell is completely described by any two of the following four properties:

- Inner radius $r_{1}$

- Outer radius $r_{2}$

- Mass $m$, one of ${ }^{239} \mathrm{Pu}$ mass $m_{239 P u},{ }^{240} \mathrm{Pu}$ mass $m_{240 P u}$, or total Pu mass $m_{P u}$ B

- Radial thickness $\Delta r$

Of these, generally only mass is acknowledged as an attribute of interest; the second property (whichever is chosen) can be considered to be a parameter of the attribute-estimation procedure, much as multiplication is a parameter necessary to accurately estimate fissile mass via most neutron measurements.

\footnotetext{
${ }^{1}$ Only $1.77 \%{ }^{240} \mathrm{Pu}$ shells were available at RFNC-VNIIEF. This is unfortunate because without variation in the relative ${ }^{240} \mathrm{Pu}$-content, it cannot be demonstrated from these measurements alone that NMIS is capable of extracting this attribute. Future joint measurements planned between the Russian Federal Nuclear Center, AllRussia Scientific Research Institute of Theoretical Physics (RFNC-VNIITF), RFNC-VNIIEF, and ORNL will demonstrate this capability through additional measurements of $10 \%-{ }^{240} \mathrm{Pu}$ shells at RFNC-VNIITF in Snezhinsk, Russia.

${ }^{2}$ V. V. Gurov, M. I. Kuvshinov, V. A. Popov, V. P. Dubinin, J. K. Mattingly, and J. T. Mihalczo, "VNIIEFORNL Joint Plutonium Measurements with NMIS and Results of Plutonium Attributes Preliminary Evaluations," July 2001.

${ }^{3}$ Due to the constant relative ${ }^{240} \mathrm{Pu}$-content of the VNIIEF shells, ${ }^{239} \mathrm{Pu}$ mass, ${ }^{240} \mathrm{Pu}$ mass, and total plutonium mass, are directly proportional to each other and are therefore equivalent measures of mass for attribute estimation purposes.
} 
In fact, multiplication is intimately related to thickness. One result of neutron diffusion theory dictates that the effective multiplication factor, $k_{\text {eff }}$, of a spherical shell is controlled by the fissile mass $m$ and geometric buckling $B$ according to

$$
k_{e f f} \sim \frac{m}{B^{2}}
$$

(" " denotes "follows") where the geometric buckling $B$ is proportional to the inverse of the radial thickness $\Delta r$ :

$$
B=\frac{\pi}{\Delta r}
$$

In other words, for any particular mass, the thicker a spherical shell, the greater its multiplication $M$ since:

$$
M=\frac{1}{1-k_{\text {eff }}}
$$

In order to account for multiplication effects in the interpretation of the passive NMIS measurements, Monte Carlo calculations were performed to estimate the unreflected $k_{\text {eff }}$ of the spherical shells listed in Table 1. The dimensions given on the diagonal are those of the existing spherical shells; the off-diagonal dimensions include all hypothetical nestings of those shells. The span of relative ${ }^{240} \mathrm{Pu}$-content was chosen to encompass both the VNIIEF $\left(\sim 2 \%-{ }^{240} \mathrm{Pu}\right)$ and the VNIITF $\left(\sim 10 \%-{ }^{240} \mathrm{Pu}\right)$ shells. The approximate relationship between total plutonium mass $m_{P u}$, radial thickness $\Delta r$, relative ${ }^{240} \mathrm{Pu}$-content $f_{240 P u}$, and multiplication factor $k_{\text {eff }}$ was estimated via least-squares regression as:

$$
k_{\text {eff }} \approx 0.034 \frac{m_{P u}^{0.156} \Delta r^{0.465}}{f_{240 P u}^{0.015}}
$$

Figure 1 compares this model for the effective multiplication factor to the calculated values listed in Table 1. Observe that over the span $1 \% \leq f_{240 P u} \leq 20 \%$ the effect on $k_{\text {eff }}$ of relative ${ }^{240} \mathrm{Pu}$-content is almost negligible. In other words, over this span multiplication is almost entirely dominated by mass and radial thickness.

Figure 2 shows a typical distribution of real coincidence acquired from a passive NMIS measurement of one of the VNIIEF plutonium-metal spherical shells. Recall that the coincidence-distribution reflects the rate of real coincidence (i.e., total coincidence less accidental coincidence) as a function of the time-delay between detectors (at zero-delay, the detectors signals are synchronized). The narrow peak about the origin arises due to coincident pairs of gammas spawned by the same chain-reaction. The broader underlying bimodal distribution arises from coincident pairs composed of one gamma and one neutron created during the same chain-reaction. Not visible in the figure is a third single-mode distribution arising from coincident pairs of neutrons spawned by the same chain-reaction; it's amplitude is such that it lies beneath the preceding two distributions.

The features of the coincidence-distribution used to estimate the multiplication parameter and mass attribute are derived from its width and integral. For this study, the distribution width 
Table 1. Monte Carlo Estimates of the Effective Multiplication Factor $k_{\text {eff }}$ for Unreflected 8-Phase Plutonium-Metal Spherical Shells

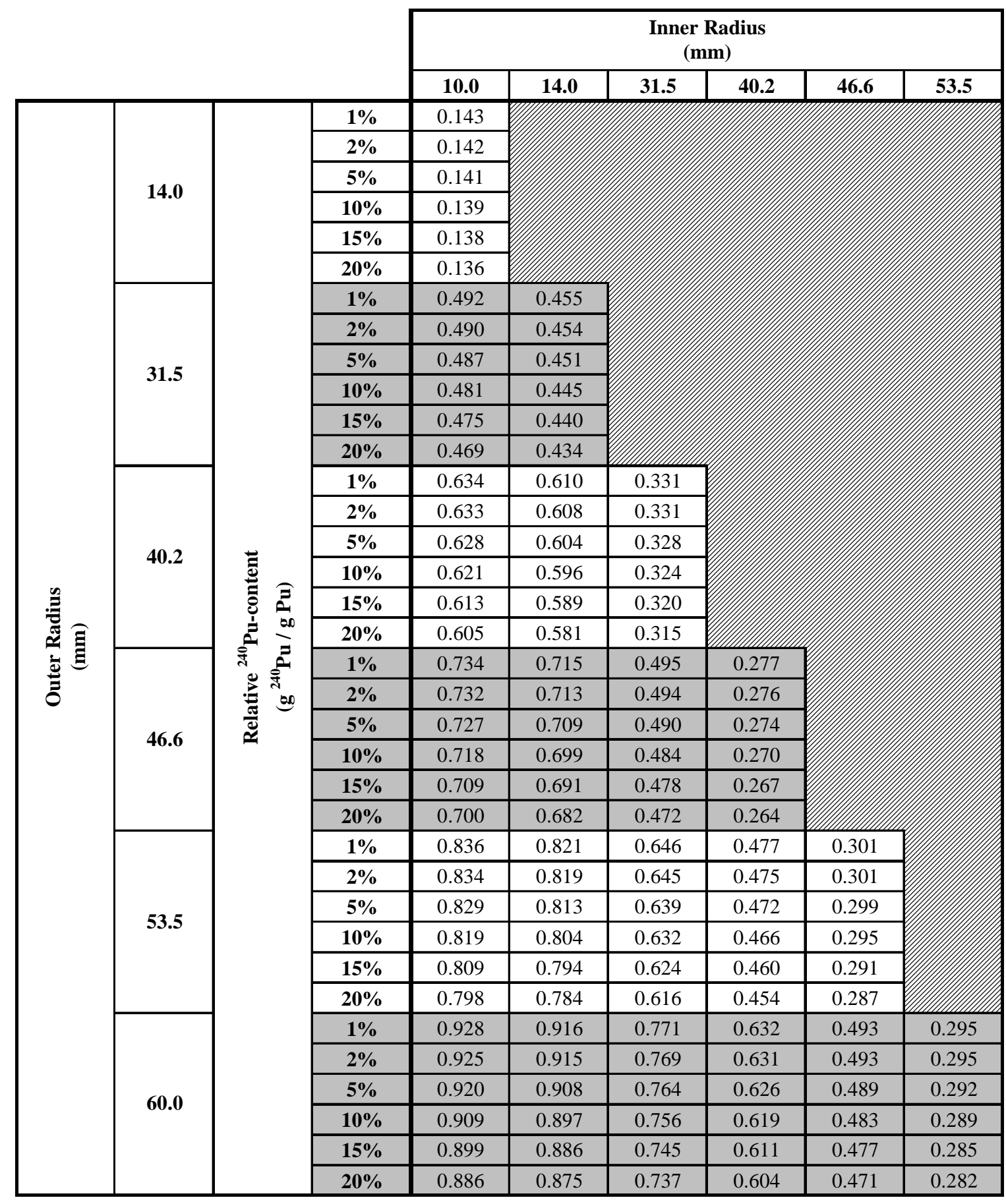




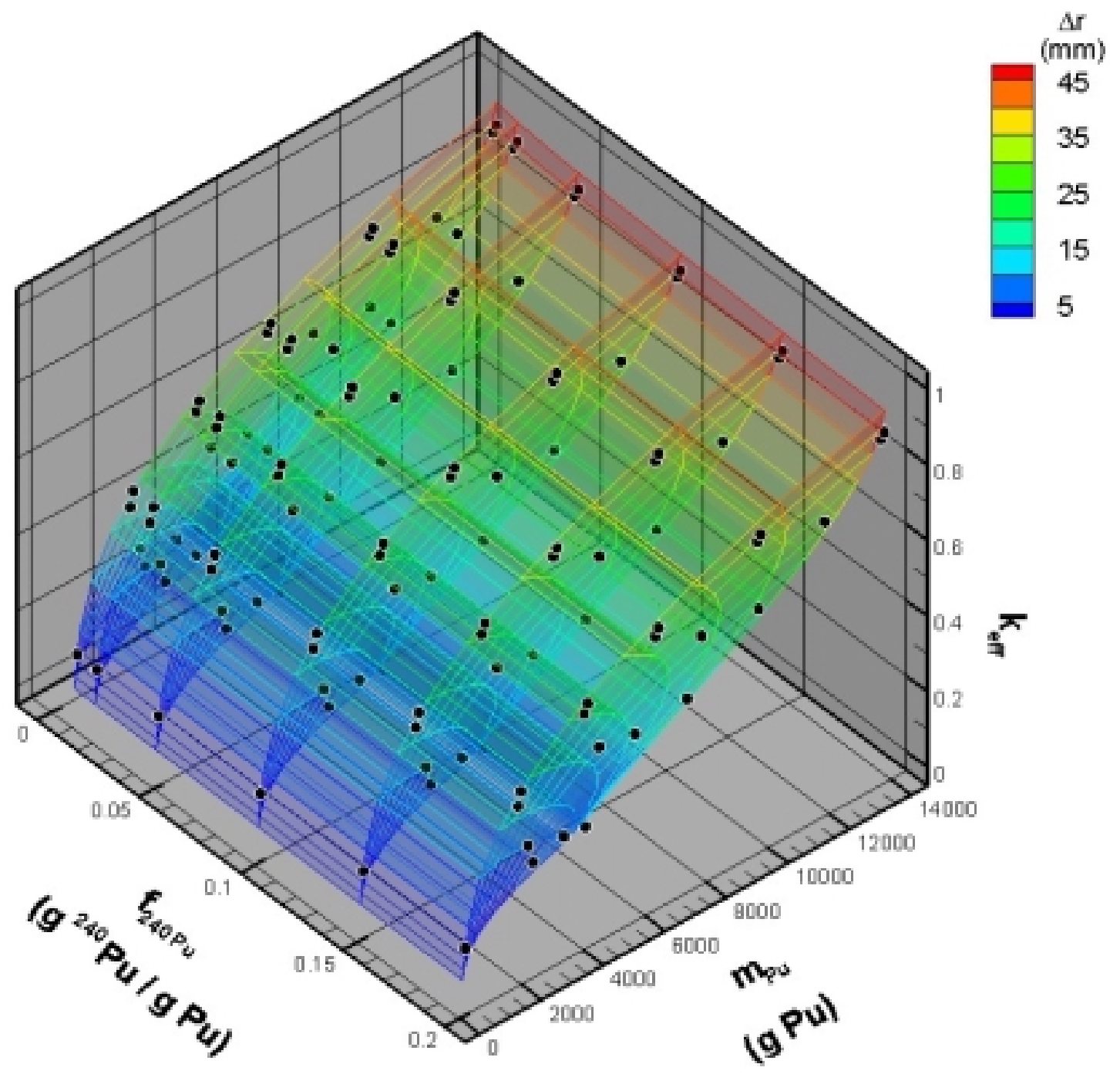

Fig. 1. Comparison of $k_{\text {eff }}$ Estimates From the Monte Carlo Calculations Listed in Table 1 to a Least-Squares Regression Power Model. The black dots represent the Monte Carlo estimates; the isosurfaces versus total plutonium mass $m_{P u}$, radial thickness $\Delta r$, relative ${ }^{240} \mathrm{Pu}$-content $f_{240 P u}$ represent the regression model. 


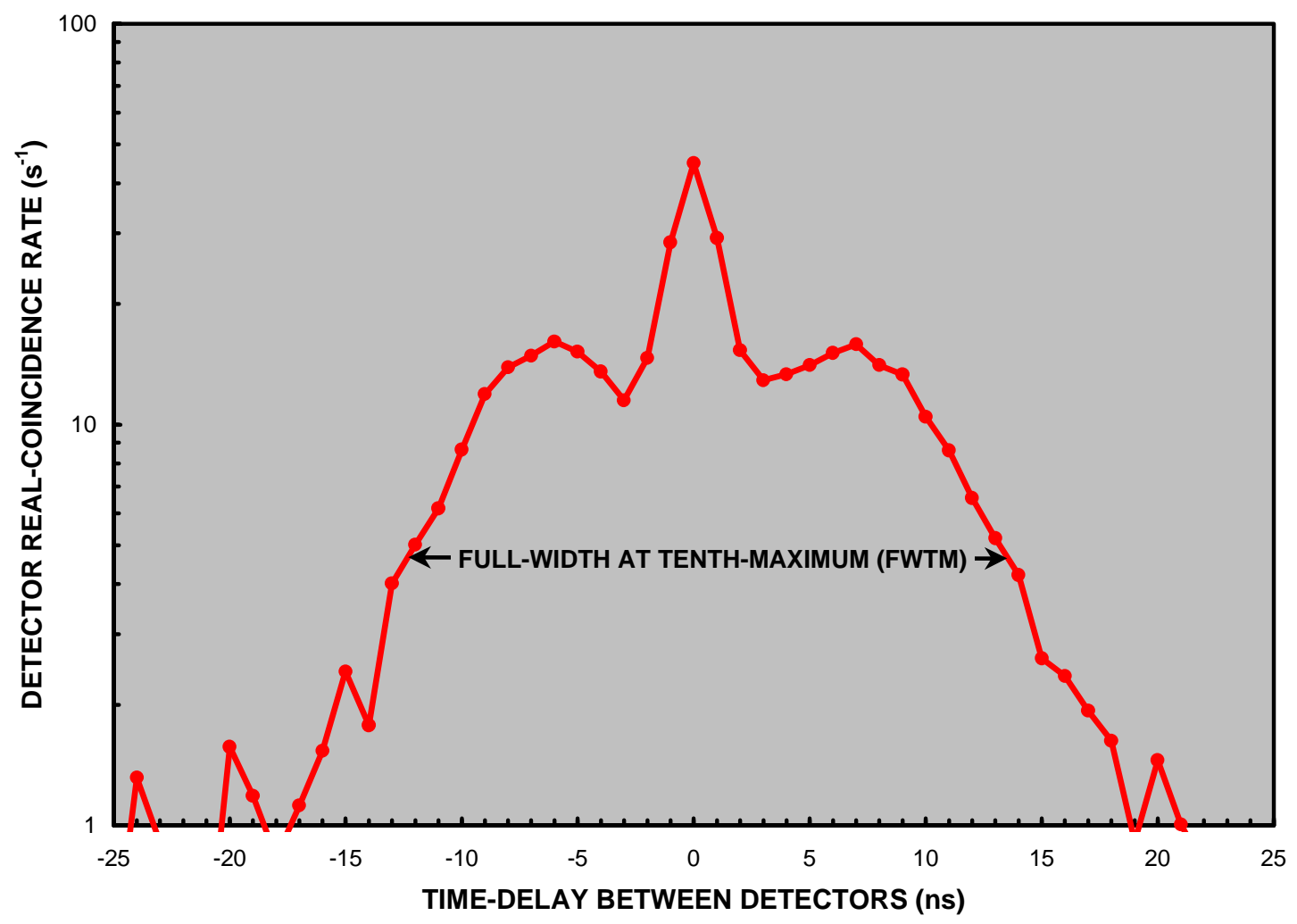

Fig. 2. A Typical Coincidence-Distribution Acquired From a Passive NMIS

Measurement of a VNIIEF Plutonium-Metal Spherical Shell. The plutonium mass and radial thickness of the specific shell measured to obtain this distribution was $3316 \mathrm{~g}$ and $6.9 \mathrm{~mm}$, respectively (inner and outer radius: $46.6 \mathrm{~mm}$ and $53.5 \mathrm{~mm}$, respectively). The features used for attribute-estimation were the distribution's full-width at tenth-maximum (FWTM) and it's FWTM area, i.e., the integral spanning the FWTM. 
was taken as its full-width at tenth-maximum, or FWTM. The distribution's integral was taken as the area spanning the FWTM, a.k.a., its FWTM area. Subsequently, the distributions width and integral will respectively be denoted by $W_{F W T M}$ and $A_{F W T M}$.

Figures 3 and 4 illustrate the dependence of the width and integral features upon multiplication and mass. The width tends to grow with increasing multiplication following a logarithmic trend, and the integral tends to grow with the product of multiplication and mass following a power rule. Least-squares regression quantifies these observations. The width feature is approximately proportional to multiplication according to

$$
W_{F W T M} \approx 12.53 \ln M+22.13
$$

and the integral feature is approximately proportional to the product of multiplication and mass according to

$$
A_{F W T M} \approx 2.37\left(M \cdot m_{240 P u}\right)^{1.14}
$$

This forward model ((1) and (2)) is readily solved to yield the inverse model that estimates multiplication and mass given the width and integral features:

$$
\begin{gathered}
M \approx \exp \left(\frac{W_{F W T M}-22.13}{12.53}\right) \\
m_{240 P u} \approx\left(\frac{A_{F W T M}}{2.37}\right)^{1 / 1.14} \exp \left(-\frac{W_{F W T M}-22.13}{12.53}\right)
\end{gathered}
$$

Substituting an observed width and integral feature into the preceding inverse model yields an estimate of multiplication and mass. The inverse model applied to the width and integral features observed for all the VNIIEF shells measured yields the estimates shown in Figs. 5 and 6. Note that in a root-mean-square (RMS) sense, the inverse model estimates multiplication to within $5 \%$ of its actual value and mass to within $7 \%$ of its actual value.

In its application to an unknown plutonium-metal spherical shell, the FWTM and FWTM area of the coincidence-distribution acquired from a passive NMIS measurement would be substituted into the inverse model to obtain an estimate of the ${ }^{240} \mathrm{Pu}$ mass according to (3b). If the unknown shell falls within the span of multiplication and mass shown in Figs. 5 and 6 then the relative error of the estimated ${ }^{240} \mathrm{Pu}$ mass will be on the order of $10 \%$. With continued development of these methods, the error may be reduced. 


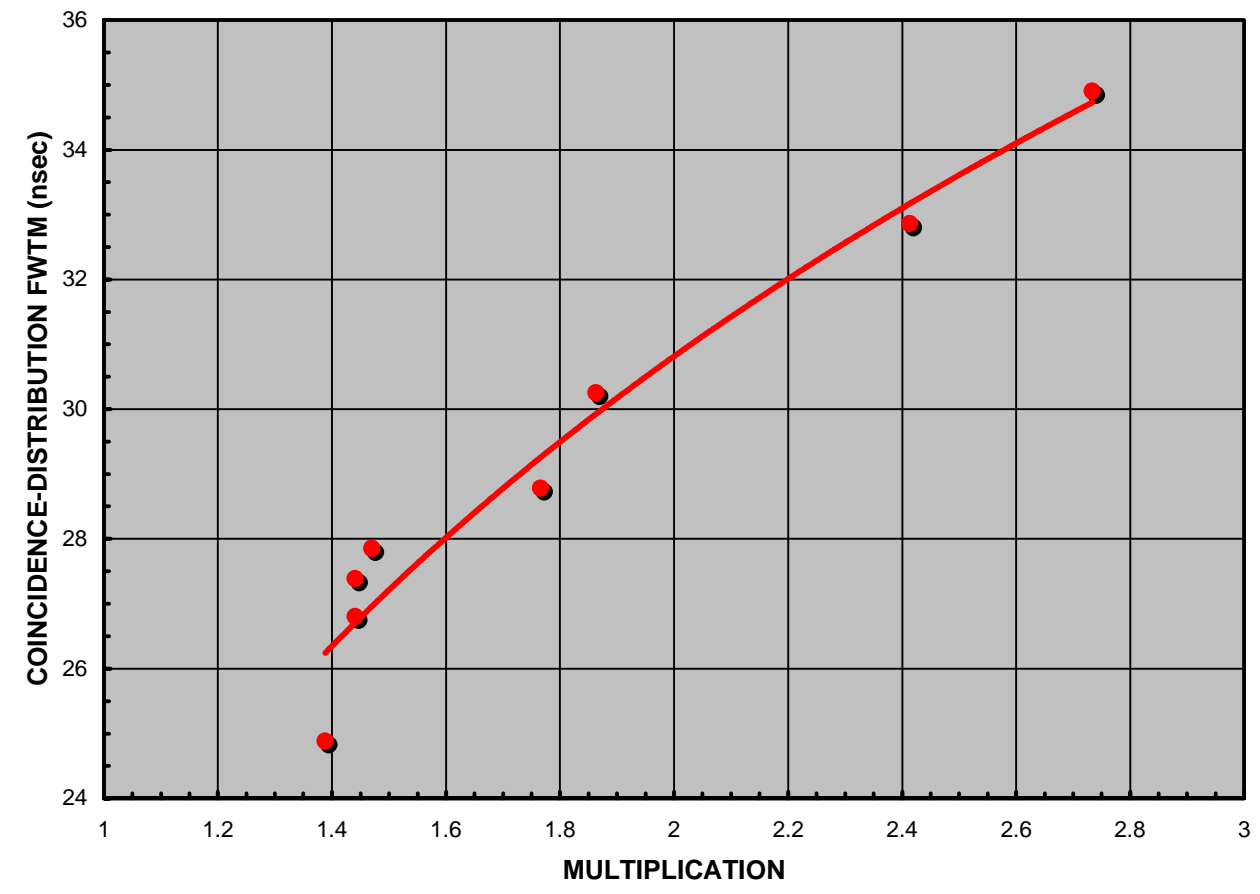

Fig. 3. Dependence of the Width Feature Upon Multiplication

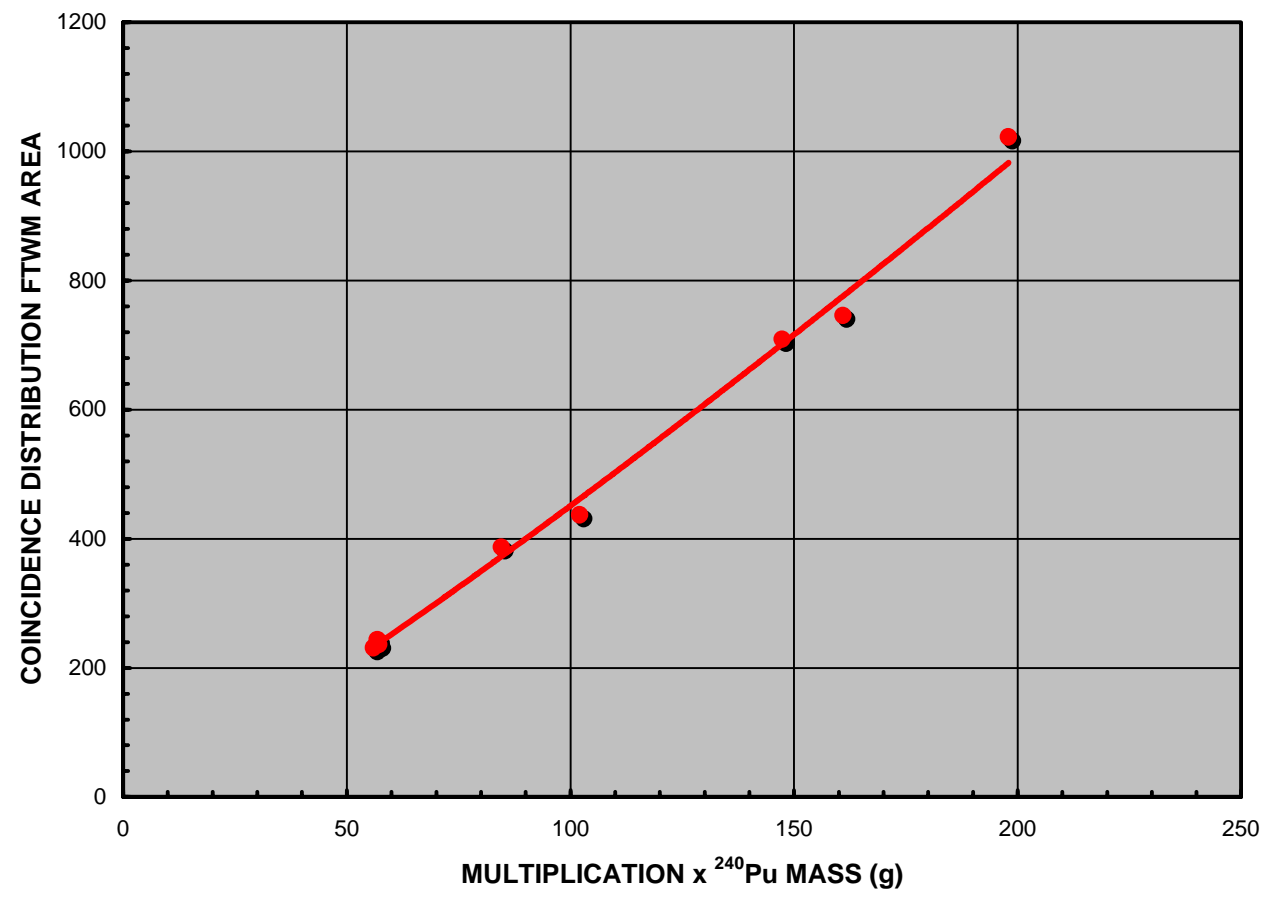

Fig. 4. Dependence of the Integral Feature on the Product of Multiplication and Mass 


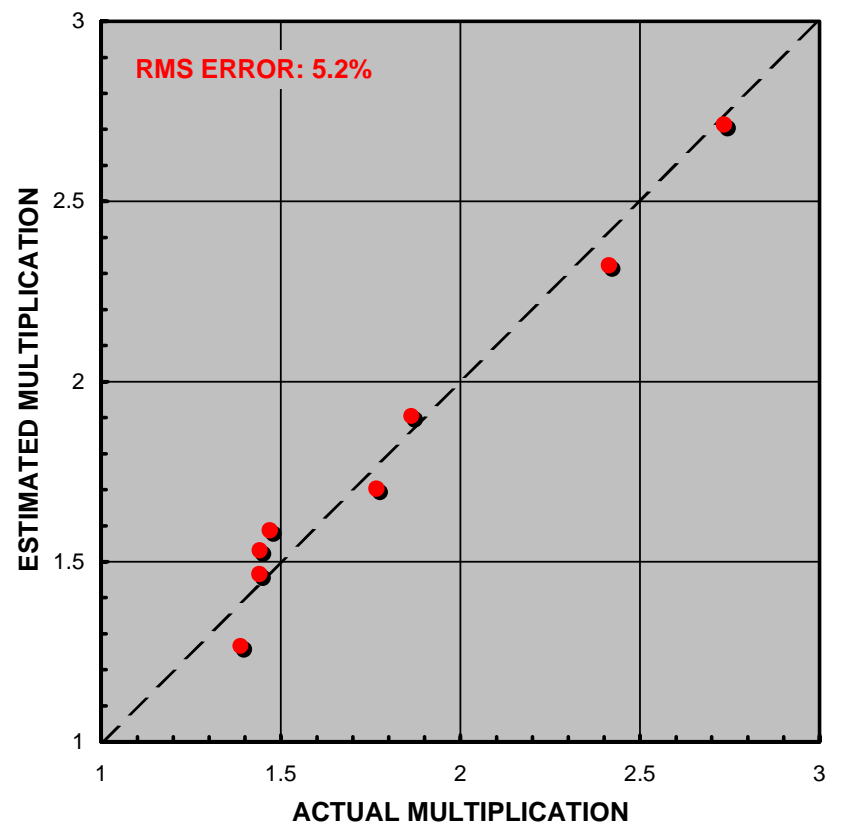

Fig. 5. Comparison of Multiplication Estimates Obtained From the Inverse Model to the Actual (i.e., Monte Carlo Calculated) Multiplication of Each VNIIEF Shell Measured

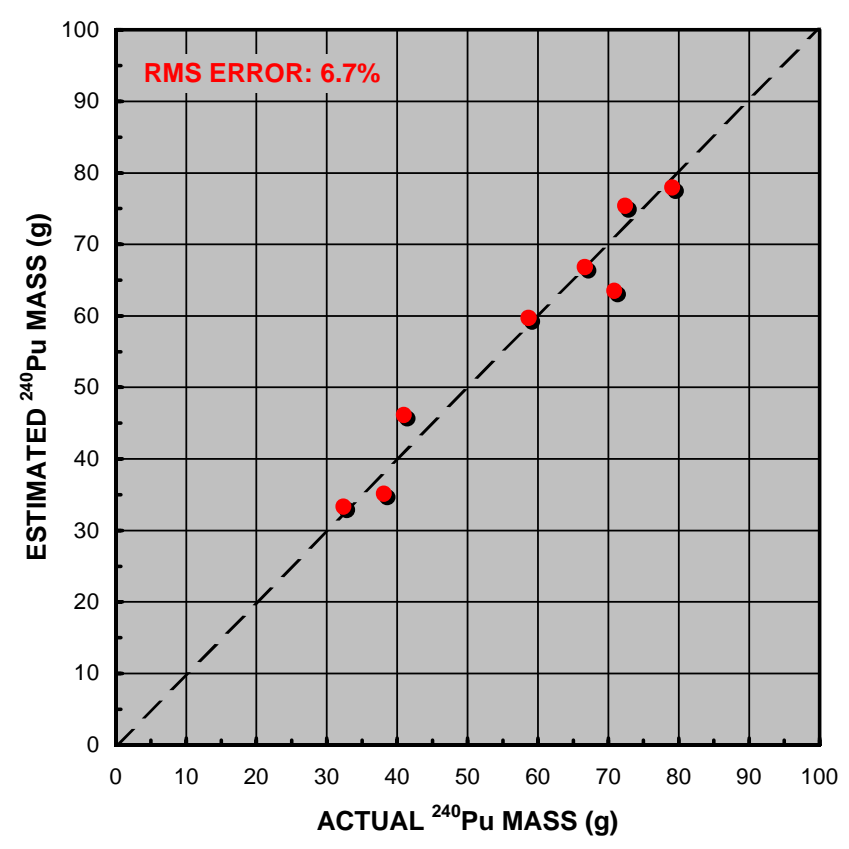

Fig. 6. Comparison of ${ }^{240} \mathrm{Pu}$ Mass Estimates Obtained From the Inverse Model to the Actual ${ }^{240}$ Pu Mass of Each VNIIEF Shell Measured 